\title{
Calf Circumference as a Screening Tool for Cognitive Frailty in Community-Dwelling Older Adults: The Korean Frailty and Aging Cohort Study (KFACS)
}

\author{
Miji Kim ${ }^{1,+}{ }^{+}$, Min Jeong Jeong ${ }^{2,+}$, Jinho Yoo ${ }^{3}\left(\mathbb{D}\right.$, Da Young Song ${ }^{2}$ and Chang Won Won ${ }^{3, * \mathbb{C}}$ \\ 1 Department of Biomedical Science and Technology, College of Medicine, East-West Medical Research \\ Institute, Kyung Hee University, Seoul 02447, Korea; mijiak@khu.ac.kr \\ 2 Elderly Frailty Research Center, Kyung Hee University, Seoul 02447, Korea; minjj914@khu.ac.kr (M.J.J.); \\ hanasdy@naver.com (D.Y.S.) \\ 3 Elderly Frailty Research Center, Department of Family Medicine, College of Medicine, \\ Kyung Hee University, Seoul 02447, Korea; jhyoo153@naver.com \\ * Correspondence: chunwon62@naver.com; Tel.: +82-2-958-8700; Fax: +82-2-958-8699 \\ + These authors contributed equally to this work.
}

Received: 30 August 2018; Accepted: 4 October 2018; Published: 8 October 2018

\begin{abstract}
The aim of this study was to examine calf circumference in relation to cognitive frailty in community-dwelling older adults. Cross-sectional analysis was performed on the first-year baseline data of 1559 adults aged 70-84 years enrolled in the Korean Frailty and Aging Cohort Study. The final analysis included 1221 adults who were non-dependent in terms of instrumental activities of daily living, who underwent frailty and cognitive function assessments. Physical frailty was defined using the Fried Frailty Index. Cognitive impairment was defined as a score 1.5 standard deviations below the age-, sex- and education-matched norms on any of four cognitive-function tests. The prevalence of cognitive frailty was $2.8 \%$ for men and $3.8 \%$ for women. After adjusting for potential confounders, in comparison to the "physically robust without cognitive impairment" group, the estimates of increased odds ratios (ORs) for low calf circumference $(<32 \mathrm{~cm})$ were much greater in the prefrail with cognitive impairment (OR 4.62, 95\% confidence interval (CI): 2.02-10.61) and frail with cognitive impairment (OR 10.94, 95\% CI: 2.87-41.68) groups in men but not in women. Low calf circumference was strongly related to cognitive frailty in men only, suggesting calf circumference can be used as an indicator of these outcomes.
\end{abstract}

Keywords: calf circumference; cognitive frailty; frailty; aging; cohort study

\section{Introduction}

Frailty is common among people with the geriatric syndrome and results in adverse health outcomes including hospitalization, institutionalization, falls, functional disability, and mortality [1,2]. Adding cognitive impairment to the operational definition of the frail phenotype could improve its predictive validity with regard to adverse health outcomes [3]. Cognitive frailty is defined as the simultaneous presence of physical frailty and cognitive impairment [4]. In the Frailty Operative Definition Consensus Conference Project, experts agreed on the importance of a more comprehensive definition of frailty, to include both physical performance and cognition components [5]. Preclinical cognitive impairment was associated with earlier-onset frailty [6], with the two conditions being closely interrelated [7]. Physical frailty showed a relationship with an increased prevalence of cognitive impairment, and co-existing physical frailty and cognitive impairment conferred a greater risk of incident dementia [8]. A longitudinal, population-based study found that reversible cognitive frailty was a short- and long-term predictor of all-cause mortality and overall dementia in non-demented 
older individuals [9]. Hence, it is important that the tools potentially useful for identifying cognitively frail individuals capture the cognitive-frailty relationship, to inform future preventive and therapeutic strategies in older adults who may progress to dementia.

Loss of skeletal muscle mass and size, which occurs with aging, is a key component in the diagnosis of sarcopenia [10] and is associated with both physical frailty and cognitive impairment [11-13]. Calf circumference has been used as a simple proxy for skeletal muscle mass, sarcopenia, and nutritional status in clinical and community settings [14-18]. Calf circumference is related to frailty and functional performance [19] and is also a significant predictor of cognitive function [20]. Furthermore, calf circumference is a useful screening tool, being a simple, convenient and non-invasive measure. Calf circumference may be more closely associated with cognitive frailty than physical frailty and cognitive impairment independently, and thus it may be useful as a marker of cognitive frailty in older adults.

The aim of this study was to examine calf circumference in relation to cognitive frailty and to determine whether calf circumference can be used to screen for physical and cognitive frailty in community-dwelling older adults enrolled in the Korean Frailty and Aging Cohort Study (KFACS).

\section{Materials and Methods}

\subsection{Study Population}

The KFACS aims to identify diverse risk and preventive factors for the progression (and associated adverse outcomes) of frailty in community-dwelling older adults. The KFACS is a Korean multicenter longitudinal study, in which the baseline survey was conducted in 2016-2017. Sex- and age-stratified community residents aged 70 to 84 years, drawn from 10 medical centers in urban and rural regions nationwide, were eligible for participation in the study [21]. In total, there were 3014 participants in the baseline survey. Our analyses were performed using the baseline (i.e., 2016) data of 1559 KFACS participants. The final analysis included 1221 participants (570 men and 651 women), after excluding 288 participants who were dependent on others for instrumental activities of daily living (IADL) and 50 participants who had missing data for the outcomes of interest, such as frailty and cognitive impairment (Figure 1). The IADL were measured across 10 domains using the Korean IADL (K-IADL) instrument: decorating, housework, preparing meals, laundry, short outings, using transportation, shopping, handling money, using the telephone, and taking medicine.

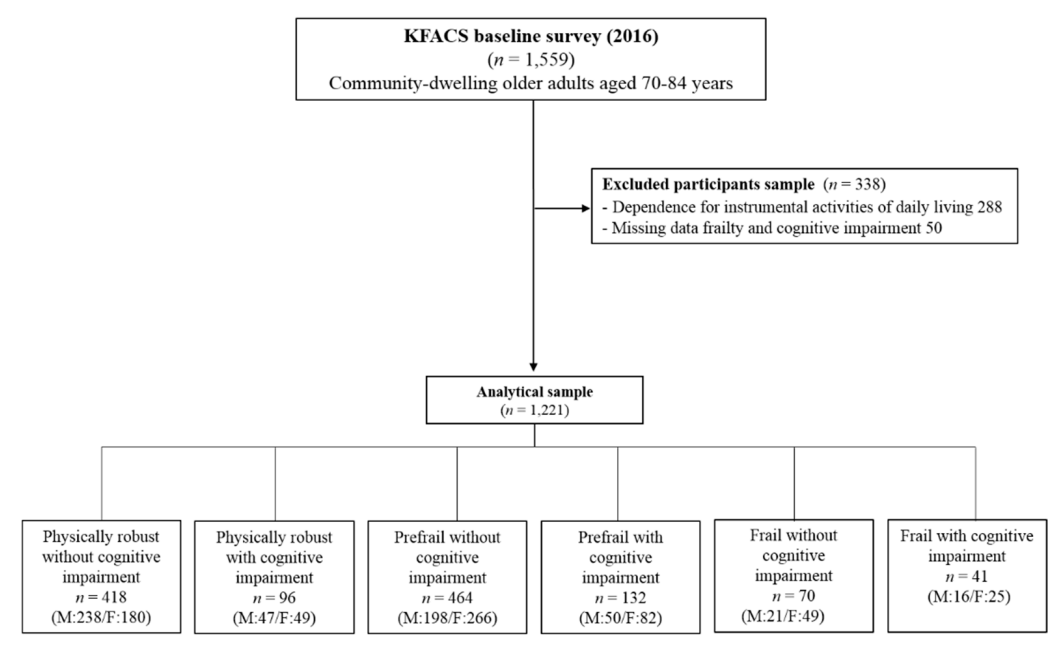

Figure 1. Flow chart of study participant selection.

\subsection{Physical Frailty, Cognitive Impairment, and Cognitive Frailty}

Physical frailty was defined using a modified version of the Fried Frailty Index, which covers five components of frailty [1]: 
1. Unintentional weight loss: responding "yes" to the question: "In the last year, have you lost more than $4.5 \mathrm{~kg}$ unintentionally?" or exhibiting unintentional weight loss $\geq 5 \%$ of total body weight in the last year.

2. Weakness: maximal grip strength $<26 \mathrm{~kg}$ for men and $<18 \mathrm{~kg}$ for women, measured twice for each hand using a digital hand grip dynamometer (T.K.K. 5401; Takei Scientific Instruments Co, Ltd., Tokyo, Japan).

3. Self-reported exhaustion: responding "yes" to either of the following statements from the Center for Epidemiological Studies-Depression scale: "I felt that everything I did was an effort" and "I could not get going" on 3 or more days per week.

4. Slowness: 4-m gait speed $<1.0 \mathrm{~m} / \mathrm{s}$, measured using an automatic timer (Gaitspeedometer Ver.1, Dynamicphysiology, Daejeon, Korea), with acceleration and deceleration phases of $1.5 \mathrm{~m}$ each. Gait speed was measured twice, and the mean values were used in the analysis.

5. Low physical activity: energy expenditure estimates (kcal/week) were calculated for various activities, and metabolic equivalent scores were derived using the International Physical Activity Questionnaire. Low physical activity level was defined as $<494.65 \mathrm{kcal}$ for men and $<283.50 \mathrm{kcal}$ for women, with these values corresponding to $20 \%$ of the total energy consumed in a population-based Korean survey of older adults from among the general population [22].

Total frailty scores (range: $0-5$ ) were calculated by allocating a score of 1 to positive responses on each of the above five components. Participants with a score of 0 were classified as "robust", a score of $1-2$ as "prefrail", and a score of 3-5 as "frail".

Cognitive impairment was defined as a score 1.5 standard deviations (SDs) below the age-, sex- and education-matched Korean norms on any one of four cognitive-function tests: The Trail Making Test, Frontal Assessment Battery, Digit Span Backward, and the Word List Recall test [23]. Cognitive function was assessed using the Korean Version of the Consortium to Establish a Registry for Alzheimer's Disease Assessment Packet [24,25], and the Korean version of the Frontal Assessment Battery [26]. The International Academy on Nutrition and Aging and the International Association of Gerontology and Geriatrics group proposed the definition of cognitive impairment using the Clinical Dementia Rating (global CDR score of 0.5), excluding concurrent dementia [4]. Because the CDR is not often available in epidemiologic studies and is difficult to implement, an alternative is required. Furthermore, mild cognitive impairment (MCI) may not be an appropriate component in the diagnostic criteria for cognitive frailty due to the lack of benefits for dementia prevention [27]. In general, scores on neuropsychological tests for individuals with $\mathrm{MCI}$ are 1.0 to $1.5 \mathrm{SDs}$ below the mean for age- and education-matched control subjects [28]. A consensus from the Subjective Cognitive Decline Initiative Working Group proposed research criteria for cognitive-function testing for pre-MCR subjective cognitive decline, which was defined as less than or equal to 1.5 standard deviations from age-, sex-, and education-adjusted norms on standardized cognitive tests $[29,30]$. In recent years, epidemiologic studies have used this definition of cognitive impairment [31,32].

Cognitive frailty was defined as the presence of both physical frailty and cognitive impairment. Based on the degree of physical frailty and cognitive impairment, participants were classified into one of six groups: physically robust without cognitive impairment, physically robust with cognitive impairment, prefrail without cognitive impairment, prefrail with cognitive impairment, frail without cognitive impairment, or frail with cognitive impairment. The percentages of the participants in each group were $34.2 \%, 7.9 \%, 38.0 \%, 10.8 \%, 5.7 \%$, and $3.4 \%$, respectively (Figure 1 ).

\subsection{Calf Circumference}

Calf circumference was measured with the participant standing upright and the legs slightly apart. A measuring tape was positioned around the calf at the point of maximum circumference; subcutaneous tissue was not compressed [15]. Our previous study suggested a cut-off value of $32 \mathrm{~cm}$ for diagnosing low muscle mass and sarcopenia based on the Asian Working Group for Sarcopenia definition for community-dwelling older adults [15]. 


\subsection{Other Measurements}

An in-person interview and health examination were performed. Participants provided information on smoking status, alcohol consumption, education level, marital status, and medical history and treatment, via a questionnaire. Comorbid status was determined by the presence of at least one of the following diseases: myocardial infarction, congestive heart failure, peripheral vascular disease, cerebrovascular disease, chronic obstructive pulmonary disease, rheumatoid arthritis, ulcers, mild liver disease, diabetes mellitus, diabetes with complications, hemiplegia, moderate or severe renal disease, any tumor, moderate or severe liver disease, leukemia, lymphoma, or acquired immune deficiency syndrome. Body mass index (BMI) was calculated as weight $(\mathrm{kg})$ divided by height squared $\left(\mathrm{m}^{2}\right)$. Body composition was measured using dual energy X-ray absorptiometry (Lunar; GE Healthcare, Madison, WI, USA; and Hologic DXA; Hologic Inc., Bedford MA, USA) and bioelectrical impedance analysis (InBody 720; InBody Co., Ltd., Seoul, Korea and X-SCAN PLUS II; Jawon Medical Inc., Seoul, Korea). Appendicular skeletal muscle mass (ASM; kg), that is, the sum of the lean soft-tissue mass in both extremities, was measured. The ASM index was calculated as the ASM divided by height squared $\left(\mathrm{m}^{2}\right)$. The definition of low muscle mass used herein was that of the Asian Working Group for Sarcopenia: $<7.0 \mathrm{~kg} / \mathrm{m}^{2}$ for men and $<5.4 \mathrm{~kg} / \mathrm{m}^{2}$ for women on dual energy X-ray absorptiometry and $<7.0$ for men and $<5.7 \mathrm{~kg} / \mathrm{m}^{2}$ for women on bioelectrical impedance analysis [33]. The Short Physical Performance Battery (SPPB) consists of three standing balance measures (tandem, semi-tandem, and side-by-side stands), five chair-stand time measures, and assessment of typical gait speed. Each test is scored from 0 to 4 , based on the normative scores of the Established Population for Epidemiologic Studies of the Elderly [34]. The scores are summed to obtain a total score, ranging from 0 to 12 [34,35]. In the Timed Up And Go (TUG) test, participants were asked to rise from an armchair of standard height; a 3-m distance was marked on the floor in front of the chair [36]. The starting position was sitting with the hands resting on the arms of the chair. The participants crossed the line before turning around and walking back to, and sitting down on, the chair. They were instructed to perform the TUG test at their own comfortable and safe walking pace. The TUG test started as soon as the participant's back ceased to be in contact with the back of the chair, and stopped when their buttocks recontacted the seat of the chair.

\subsection{Ethics}

The KFACS protocol was approved by the Institutional Review Board (IRB) of the Clinical Research Ethics Committee of Kyung Hee University Medical Center, Seoul, Korea, and all participants provided written informed consent (IRB number: 2015-12-103). This study was exempt from review by the IRB (IRB number: 2018-05-095).

\subsection{Statistical Analysis}

The data are presented as means \pm SD or as numbers (percentages). The relationships among participant characteristics and frailty and cognitive function indices were analyzed by analysis of variance (ANOVA) and chi-square tests. We calculated geometric means (standard error), estimates, and $95 \%$ confidence intervals (CIs) using a generalized linear model to assess calf circumference according to physical frailty and cognitive impairment status. In the secondary analysis, we used multivariable logistic regression models with Firth's penalized likelihood method to address issues of small sample size [37]. Odds ratios $(95 \% \mathrm{CIs})$ for low calf circumference $(<32 \mathrm{~cm})$ were estimated by cognitive frailty status. Models were adjusted for age (continuous), total alcohol consumption (never or ever drinker), years of education ( $0-6$ or $\geq 7$ ), number of comorbidities ( 0 or $\geq 1)$, number of medications $(0,1-4$, or $\geq 5)$, and body mass index $(\leq 23,23-24.9,25-26.9, \geq 27)$. All analyses were conducted using SPSS software (ver. 23.0; SPSS Inc., Chicago, IL, USA) and SAS version 9.4 (SAS Institute, Inc., Cary, NC, USA). Significance was set at a two-sided $p$-value of $<0.05$. 


\section{Results}

Table 1 presents the baseline characteristics of the male participants according to physical frailty and cognitive impairment status. The mean age of the men was 76.4 years; $2.8 \%$ were both physically frail and cognitively impaired, that is, cognitively frail. Compared with the physically robust without cognitive impairment group, those who were prefrail or frail, with or without cognitive impairment, were older, less educated, less likely to be married and had a smaller calf circumference. Table 2 presents the baseline characteristics of the female participants. The mean age of the women was 75.7 years; $3.8 \%$ were cognitively frail, and $12.6 \%$ were cognitively prefrail.

Tables 3 and 4 show the generalized linear model estimates and 95\% CIs for calf circumference according to physical frailty and cognitive impairment status in men and women. After adjusting for potential confounders, including age, sex, alcohol consumption, years of education, marital status, comorbidities, medications, and body mass index (Model 3), significant decreases in calf circumference were found for the physically robust with cognitive impairment group (estimate $(\beta)=-0.682,95 \%$ $C I=-1.352 ;-0.031)$, the prefrail without cognitive impairment group $(\beta=-0.745,95 \% C I=-1.150$; $-0.340)$, the prefrail with cognitive impairment group $(\beta=-0.932,95 \% C I=-1.589 ;-0.275)$, the frail without cognitive impairment group $(\beta=-1.229,95 \% \mathrm{CI}=-2.274 ;-0.324)$, and the frail with cognitive impairment group $(\beta=-2.471,95 \% \mathrm{CI}=-3.566 ;-1.377)$, as compared with the physically robust without cognitive impairment group (all <0.05). In women (Model 3), significant decreases were found in calf circumference for the prefrail without cognitive impairment group $(\beta=-0.408$, $95 \% \mathrm{CI}=-1.806 ;-0.011)$, the prefrail with cognitive impairment group $(\beta=-0.681,95 \% \mathrm{CI}=-1.241$; $-0.121)$, and the frail without cognitive impairment group $(\beta=-1.104,95 \% \mathrm{CI}=-1.799 ;-0.409)$ as compared with the physically robust without cognitive impairment group (all <0.05). However, the physically robust with cognitive impairment and frail with cognitive impairment groups did not show significant associations with decreased calf circumference.

Figure 2 shows that the geometric mean calf circumference after adjusting for potential confounding variables (Model 3) showed a significant decreasing trend across the six groups, from the physically robust without cognitive impairment group to the frail with cognitive impairment group in men $(35.2,34.5,34.4,34.2,33.9,32.7$, respectively, $p$ for trend $<0.001)$. In women, this pattern was not seen $(33.1,33.1,32.7,32.4,32.0,32.6$, respectively, $p$ for trend $=0.018)$. In the post-hoc analysis, the geometric mean calf circumference of the men was significantly lower in the frail with cognitive impairment group compared with the physically robust without cognitive impairment, physically robust with cognitive impairment, prefrail without cognitive impairment, and prefrail with cognitive impairment groups (all $p<0.5$ ); this pattern was not seen in women. Figure 3 shows the association between low calf circumference $(<32 \mathrm{~cm})$ and cognitive frailty status. The percentages with a low calf circumference were $19.5 \%$ for mean and $37.6 \%$ for women. The odds ratios (ORs) for low calf circumference are presented by cognitive frailty status. In comparison to the "physically robust without cognitive impairment" group, the estimates of increased ORs for low calf circumference were much greater in the prefrail with cognitive impairment and frail with cognitive impairment groups in men. After adjusting for potential confounding variables (Model 3), the ORs (95\% CI) were 1.69 (0.58-4.94) for physically robust with cognitive impairment, 2.44 (1.36-4.37) for prefrail without cognitive impairment, 4.62 (2.02-10.61) for prefrail with cognitive impairment, 2.66 (0.81-8.68) for frail without cognitive impairment, and 10.94 (2.87-41.68) for frail with cognitive impairment. However, there were no associations between low calf circumference and cognitive frailty status in women. When compared with the physically robust without cognitive impairment reference group, after controlling for potential confounders (Model 3), the ORs (95\% CI) were 1.04 (0.48-2.22) for physically robust with cognitive impairment, 1.30 (0.80-2.11) for prefrail without cognitive impairment, 1.93 (1.00-3.71) for prefrail with cognitive impairment, $1.83(0.82-4.11)$ for frail without cognitive impairment, and 1.26 (0.45-3.51) for frail with cognitive impairment. 
Table 1. Characteristics of the male participants $(n=570)$ according to physical frailty and cognitive impairment status.

\begin{tabular}{|c|c|c|c|c|c|c|c|c|c|c|c|c|c|}
\hline \multirow{2}{*}{$\begin{array}{c}\text { Variable } \\
\text { Age (years) }\end{array}$} & \multicolumn{2}{|c|}{$\begin{array}{l}\text { Physically Robust } \\
\text { without Cog Imp } \\
\qquad(n=238)\end{array}$} & \multicolumn{2}{|c|}{$\begin{array}{l}\text { Physically Robust } \\
\text { with Cog Imp } \\
\text { ( } n=47)\end{array}$} & \multicolumn{2}{|c|}{$\begin{array}{l}\text { Prefrail without } \operatorname{Cog} \\
\quad \operatorname{Imp}(n=198)\end{array}$} & \multicolumn{2}{|c|}{$\begin{array}{l}\text { Prefrail with Cog } \\
\quad \operatorname{Imp}(n=50)\end{array}$} & \multicolumn{2}{|c|}{$\begin{array}{l}\text { Frail without Cog } \\
\quad \operatorname{Imp}(n=21)\end{array}$} & \multicolumn{2}{|c|}{$\begin{array}{l}\text { Frail with Cog Imp } \\
\qquad(n=16)\end{array}$} & \multirow{2}{*}{$\begin{array}{c}p \text {-Value } \\
<0.001\end{array}$} \\
\hline & 75.7 & (3.7) & 76.1 & (3.7) & 76.7 & (4.1) & 77.4 & (3.6) & 78.8 & $(3.8)$ & 78.3 & (3.6) & \\
\hline BMI $\left(\mathrm{kg} / \mathrm{m}^{2}\right)$ & 24.0 & (2.7) & 25.0 & (2.4) & 23.9 & (3.1) & 23.9 & (3.1) & 23.2 & (3.6) & 21.2 & (2.5) & 0.001 \\
\hline \multicolumn{14}{|l|}{ Smoking status } \\
\hline Never & 50 & $(21.0)$ & 15 & $(31.9)$ & 37 & $(18.7)$ & 10 & $(20.0)$ & 6 & $(28.6)$ & 2 & $(12.5)$ & 0.363 \\
\hline Ever & 188 & $(79.0)$ & 32 & $(68.1)$ & 161 & $(81.3)$ & 40 & $(80.0)$ & 15 & $(71.4)$ & 14 & $(87.5)$ & \\
\hline \multicolumn{14}{|l|}{ Alcohol (servings per week) ${ }^{a}$} \\
\hline Never & 62 & (26.1) & 15 & (32.6) & 68 & (34.3) & 25 & $(50.0)$ & 9 & $(42.9)$ & 7 & $(43.8)$ & 0.018 \\
\hline Ever & 176 & $(74.0)$ & 31 & $(67.4)$ & 130 & $(65.7)$ & 25 & $(50.0)$ & 12 & $(57.1)$ & 9 & $(56.2)$ & \\
\hline \multicolumn{14}{|l|}{ Education (years) } \\
\hline $0-6$ & 56 & (23.5) & 11 & (23.4) & 62 & $(31.3)$ & 16 & $(32.0)$ & 11 & $(52.4)$ & 8 & $(50.0)$ & 0.016 \\
\hline$\geq 7$ & 182 & $(76.5)$ & 36 & (76.6) & 136 & $(68.7)$ & 34 & $(68.0)$ & 10 & $(47.6)$ & 8 & $(50.0)$ & \\
\hline \multicolumn{14}{|l|}{ Marital status } \\
\hline Married & 218 & (91.6) & 40 & (85.1) & 172 & $(86.9)$ & 42 & $(84.0)$ & 18 & $(85.7)$ & 11 & $(68.8)$ & 0.077 \\
\hline $\begin{array}{l}\text { Unmarried, divorced, } \\
\text { widowed, or separated }\end{array}$ & 20 & $(8.4)$ & 7 & (14.9) & 26 & (13.1) & 8 & $(16.0)$ & 3 & (14.3) & 5 & $(31.2)$ & \\
\hline SPPB score ${ }^{a}$ & 11.4 & $(0.9)$ & 11.1 & (1.3) & 11.0 & $(1.2)$ & 10.6 & $(1.5)$ & 10.0 & (2.3) & 9.1 & $(2.2)$ & $<0.001$ \\
\hline \multicolumn{14}{|l|}{ Timed Up and Go test } \\
\hline$\leq 10 \mathrm{~s}$ & 165 & (69.3) & 25 & $(53.2)$ & 96 & $(48.5)$ & 17 & $(34.0)$ & 2 & $(9.5)$ & 1 & $(6.2)$ & $<0.001$ \\
\hline$>10 \mathrm{~s}$ & 73 & (30.7) & 22 & $(46.8)$ & 102 & $(51.5)$ & 33 & $(66.0)$ & 19 & $(90.5)$ & 15 & $(93.8)$ & \\
\hline ASM $/$ height $^{2}\left(\mathrm{~kg} / \mathrm{m}^{2}\right)^{\mathrm{a}}$ & 7.2 & $(0.9)$ & 7.4 & $(0.8)$ & 7.1 & $(1.0)$ & 7.2 & $(0.9)$ & 7.1 & $(1.0)$ & 6.2 & $(0.7)$ & $<0.001$ \\
\hline Normal muscle mass & 139 & $(58.9)$ & 34 & $(72.3)$ & 98 & $(49.8)$ & 27 & $(54.0)$ & 13 & $(61.9)$ & 2 & $(12.5)$ & 0.001 \\
\hline Low muscle mass & 97 & $(41.1)$ & 13 & (27.7) & 99 & $(50.2)$ & 23 & $(46.0)$ & 8 & (38.1) & 14 & (87.5) & \\
\hline \multicolumn{14}{|l|}{ Number of comorbidities } \\
\hline 0 & 160 & $(67.2)$ & 36 & $(76.6)$ & 103 & $(52.0)$ & 28 & $(56.0)$ & 6 & $(28.6)$ & 9 & $(56.2)$ & $<0.001$ \\
\hline$\geq 1$ & 78 & $(32.8)$ & 11 & (23.4) & 95 & $(48.0)$ & 22 & $(44.0)$ & 15 & $(71.4)$ & 7 & $(43.8)$ & \\
\hline \multicolumn{14}{|l|}{ Number of medications ${ }^{a}$} \\
\hline 0 & 54 & (22.7) & 12 & (25.5) & 31 & (15.7) & 4 & $(8.0)$ & 0 & $(0.0)$ & 1 & $(6.2)$ & $<0.001$ \\
\hline $1-4$ & 112 & $(47.1)$ & 26 & (55.3) & 88 & $(44.4)$ & 20 & $(40.0)$ & 7 & (33.3) & 7 & $(43.8)$ & \\
\hline$\geq 5$ & 72 & $(30.2)$ & 9 & (19.2) & 79 & (39.9) & 26 & (52.0) & 14 & $(66.7)$ & 8 & $(50.0)$ & \\
\hline Calf circumference $(\mathrm{cm})$ & 34.8 & (2.4) & 34.7 & $(2.8)$ & 33.8 & $(2.8)$ & 33.6 & $(2.8)$ & 32.6 & (3.4) & 30.5 & $(2.4)$ & $<0.001$ \\
\hline
\end{tabular}

Note: Values are means $( \pm \mathrm{SD})$ or numbers (percentages). $p$-values were calculated by ANOVA for continuous variables and by chi-square or Fisher's exact test for categorical variables.

ASM, appendicular skeletal mass; BMI, body mass index; Cog Imp, cognitive impairment; SPPB, Short Physical Performance Battery. ${ }^{a}$ missing values. 
Table 2. Characteristics of the female participants $(n=651)$ according to physical frailty and cognitive impairment status.

\begin{tabular}{|c|c|c|c|c|c|c|c|c|c|c|c|c|c|}
\hline \multirow{2}{*}{$\begin{array}{c}\text { Variable } \\
\text { Age (years) }\end{array}$} & \multicolumn{2}{|c|}{$\begin{array}{l}\text { Physically Robust } \\
\text { without Cog Imp } \\
\qquad(n=180)\end{array}$} & \multicolumn{2}{|c|}{$\begin{array}{l}\text { Physically Robust } \\
\text { with Cog Imp } \\
(n=49)\end{array}$} & \multicolumn{2}{|c|}{$\begin{array}{l}\text { Prefrail without } \operatorname{Cog} \\
\quad \operatorname{Imp}(n=266)\end{array}$} & \multicolumn{2}{|c|}{$\begin{array}{l}\text { Prefrail with Cog } \\
\quad \operatorname{Imp}(n=82)\end{array}$} & \multicolumn{2}{|c|}{$\begin{array}{l}\text { Frail without Cog } \\
\quad \operatorname{Imp}(n=49)\end{array}$} & \multicolumn{2}{|c|}{$\begin{array}{l}\text { Frail with Cog Imp } \\
\qquad(n=25)\end{array}$} & \multirow{2}{*}{$\begin{array}{c}p \text {-Value } \\
<0.001\end{array}$} \\
\hline & 74.5 & (3.4) & 75.2 & (3.6) & 75.7 & $(4.0)$ & 76.0 & (3.3) & 78.1 & $(4.3)$ & 78.5 & $(3.2)$ & \\
\hline $\operatorname{BMI}\left(\mathrm{kg} / \mathrm{m}^{2}\right)$ & 24.9 & (3.0) & 24.0 & $(2.2)$ & 24.5 & (2.9) & 25.0 & $(2.7)$ & 24.3 & (3.3) & 24.9 & (3.9) & 0.704 \\
\hline \multicolumn{14}{|l|}{ Smoking status } \\
\hline Never & 177 & $(98.3)$ & 46 & $(93.9)$ & 259 & $(97.4)$ & 81 & $(98.8)$ & 47 & $(95.9)$ & 24 & $(96.0)$ & 0.360 \\
\hline Ever & 3 & $(1.7)$ & 3 & $(6.1)$ & 7 & $(2.6)$ & 1 & $(1.2)$ & 2 & $(4.1)$ & 1 & $(4.0)$ & \\
\hline \multicolumn{14}{|l|}{ Alcohol (servings per week) ${ }^{a}$} \\
\hline Never & 103 & $(58.2)$ & 34 & $(69.4)$ & 165 & $(62.3)$ & 47 & $(58.8)$ & 38 & (77.6) & 15 & $(60.0)$ & 0.176 \\
\hline Ever & 74 & $(41.8)$ & 15 & (30.6) & 100 & $(37.7)$ & 33 & $(41.3)$ & 11 & (22.4) & 10 & $(40.0)$ & \\
\hline \multicolumn{14}{|l|}{ Education (years) } \\
\hline $0-6$ & 84 & $(46.7)$ & 29 & $(59.2)$ & 150 & $(56.4)$ & 62 & $(75.6)$ & 43 & $(87.8)$ & 21 & $(84.0)$ & $<0.001$ \\
\hline$\geq 7$ & 96 & $(53.3)$ & 20 & $(40.8)$ & 116 & $(43.6)$ & 20 & $(24.4)$ & 6 & $(12.2)$ & 4 & $(16.0)$ & \\
\hline \multicolumn{14}{|l|}{ Marital status } \\
\hline Married & 85 & $(47.2)$ & 20 & $(40.8)$ & 123 & $(46.2)$ & 30 & $(36.6)$ & 21 & $(42.9)$ & 8 & $(32.0)$ & 0.439 \\
\hline Unmarried, divorced, & 95 & $(52.8)$ & 29 & $(59.2)$ & 143 & $(53.8)$ & 52 & $(63.4)$ & 28 & $(57.1)$ & 17 & $(68.0)$ & \\
\hline SPPB score ${ }^{a}$ & 11.3 & $(1.0)$ & 11.2 & $(1.1)$ & 10.8 & $(1.2)$ & 10.3 & (1.5) & 9.4 & $(1.8)$ & 9.0 & (1.6) & $<0.001$ \\
\hline \multicolumn{14}{|l|}{ Timed Up and Go test } \\
\hline$\leq 10 \mathrm{~s}$ & 125 & $(69.4)$ & 34 & $(69.4)$ & 134 & $(50.4)$ & 25 & $(30.5)$ & 3 & $(6.1)$ & 0 & $(0.0)$ & $<0.001$ \\
\hline$>10 \mathrm{~s}$ & 55 & (30.6) & 15 & $(30.6)$ & 132 & $(49.6)$ & 57 & $(69.5)$ & 46 & $(93.9)$ & 25 & (100.0) & \\
\hline ASM $/$ height $^{2}\left(\mathrm{~kg} / \mathrm{m}^{2}\right)^{\mathrm{a}}$ & 6.1 & $(0.9)$ & 6.2 & $(0.9)$ & 6.0 & $(0.9)$ & 5.9 & $(0.8)$ & 6.0 & $(0.9)$ & 6.0 & $(1.1)$ & 0.704 \\
\hline Normal muscle mass & 141 & $(78.3)$ & 43 & $(87.8)$ & 206 & $(77.4)$ & 54 & $(65.9)$ & 33 & $(67.4)$ & 17 & $(68.0)$ & 0.034 \\
\hline Low muscle mass & 39 & $(21.7)$ & 6 & $(12.2)$ & 60 & $(22.6)$ & 28 & $(34.1)$ & 16 & $(32.7)$ & 8 & $(32.0)$ & \\
\hline \multicolumn{14}{|l|}{ Number of comorbidities } \\
\hline 0 & 124 & $(68.9)$ & 39 & $(79.6)$ & 179 & $(67.3)$ & 48 & $(58.5)$ & 27 & $(55.1)$ & 18 & $(72.0)$ & 0.079 \\
\hline$\geq 1$ & 56 & $(31.1)$ & 10 & $(20.4)$ & 87 & (32.7) & 34 & (41.5) & 22 & $(44.9)$ & 7 & $(28.0)$ & \\
\hline \multicolumn{14}{|l|}{ Number of medications ${ }^{a}$} \\
\hline 0 & 33 & $(18.3)$ & 11 & (22.5) & 29 & $(10.9)$ & 14 & $(17.1)$ & 1 & $(2.0)$ & 6 & $(24.0)$ & $<0.001$ \\
\hline $1-4$ & 107 & $(59.4)$ & 28 & $(57.1)$ & 162 & $(60.9)$ & 35 & $(42.7)$ & 19 & $(38.8)$ & 12 & $(48.0)$ & \\
\hline$\geq 5$ & 40 & $(22.2)$ & 10 & $(20.4)$ & 75 & $(28.2)$ & 33 & $(40.2)$ & 29 & $(59.2)$ & 7 & $(28.0)$ & \\
\hline Calf circumference $(\mathrm{cm})$ & 33.1 & $(2.7)$ & 32.7 & $(2.1)$ & 32.6 & $(2.6)$ & 32.3 & $(2.9)$ & 31.1 & $(3.2)$ & 31.7 & $(3.0)$ & $<0.001$ \\
\hline
\end{tabular}

Note: Values are means $( \pm \mathrm{SD})$ or numbers (percentages). $p$-values were calculated by ANOVA for continuous variables and by chi-square or Fisher's exact test for categorical variables.

ASM, appendicular skeletal mass; BMI, body mass index; Cog Imp, cognitive impairment; SPPB, Short Physical Performance Battery. ${ }^{a}$ missing values. 
Table 3. Generalized linear model estimates for calf circumference $(\mathrm{cm})$ according to physical frailty and cognitive impairment status in men.

\begin{tabular}{|c|c|c|c|c|c|c|c|c|c|c|c|c|}
\hline \multirow{2}{*}{ Variable } & \multicolumn{3}{|c|}{ Unadjusted } & \multicolumn{3}{|c|}{ Model 1} & \multicolumn{3}{|c|}{ Model 2} & \multicolumn{3}{|c|}{ Model 3} \\
\hline & Estimate & $95 \%$ CI & $p$-Value & Estimate & $95 \% \mathrm{CI}$ & $p$-Value & Estimate & $95 \%$ CI & $p$-Value & Estimate & $95 \%$ CI & $p$-Value \\
\hline Cognitive frailty status & & & & & & & & & & & & \\
\hline Physically robust without $\operatorname{Cog} \operatorname{Imp}$ & Ref. & & & Ref. & & & Ref. & & & Ref. & & \\
\hline Physically robust with Cog Imp & -0.139 & $-0.982,0.703$ & 0.746 & -0.109 & $-0.947,0.729$ & 0.789 & -1.116 & $-0.956,0.723$ & 0.786 & -0.682 & $-1.352,-0.013$ & 0.046 \\
\hline Prefrail without $\operatorname{Cog}$ Imp & -0.946 & $-1.472,-0.457$ & 0.000 & -0.885 & $-1.393,-0.377$ & 0.001 & -0.803 & $-1.310,-0.296$ & 0.002 & -0.745 & $-1.150,-0.340$ & $<0.001$ \\
\hline Prefrail with Cog Imp & -1.243 & $-2.064,-0.422$ & 0.003 & -1.107 & $-1.930,-0.285$ & 0.008 & -0.959 & $-1.783,-1.134$ & 0.023 & -0.932 & $-1.589,-0.275$ & 0.005 \\
\hline Frail without $\operatorname{Cog} \operatorname{Imp}$ & -2.182 & $-3.383,-0.980$ & 0.000 & -1.945 & $-3.152,-0.738$ & 0.002 & -1.697 & $-2.905,-0.488$ & 0.006 & -1.299 & $-2.274,-0.324$ & 0.009 \\
\hline Frail with Cog Imp & -4.301 & $-5.664,-2.938$ & 0.000 & -4.098 & $-5.462,-2.735$ & 0.000 & -3.862 & $-5.224,-2.501$ & 0.000 & -2.471 & $-3.566,-1.377$ & $<0.001$ \\
\hline
\end{tabular}

Note: CI, 95\% confidence intervals; Cog Imp, cognitive impairment; Ref., reference. Model 1: adjusted for age (years; continuous). Model 2: adjusted for age (years; continuous), alcohol consumption (never, ever), and years of education ( $0-6, \geq 7)$. Model 3: adjusted for age (years; continuous), alcohol consumption (never, ever), years of education (0-6, $\geq 7)$, comorbidities $(0, \geq 1)$, medications $(0,1-4, \geq 5)$, and body mass index $(\leq 23,23-24.9,25-26.9, \geq 27)$.

Table 4. Generalized linear model estimates for calf circumference $(\mathrm{cm})$ according to physical frailty and cognitive impairment status in women

\begin{tabular}{|c|c|c|c|c|c|c|c|c|c|c|c|c|}
\hline \multirow{2}{*}{ Variable } & \multicolumn{3}{|c|}{ Unadjusted } & \multicolumn{3}{|c|}{ Model 1} & \multicolumn{3}{|c|}{ Model 2} & \multicolumn{3}{|c|}{ Model 3} \\
\hline & Estimate & $95 \% \mathrm{CI}$ & $p$-Value & Estimate & $95 \%$ CI & $p$-Value & Estimate & $95 \% \mathrm{CI}$ & $p$-Value & Estimate & $95 \% \mathrm{CI}$ & $p$-Value \\
\hline Cognitive frailty status & & & & & & & & & & Ref. & & \\
\hline Physically robust without $\operatorname{Cog} \operatorname{Imp}$ & Ref. & & & Ref. & & & Ref. & & & & & \\
\hline Physically robust with $\operatorname{Cog} \operatorname{Imp}$ & -0.370 & $-1.216,0.476$ & 0.391 & -0.282 & $-1.116,0.551$ & 0.507 & -0.232 & $-1.071,0.606$ & 0.587 & 0.021 & $-1.636,0.677$ & 0.951 \\
\hline Prefrail without $\operatorname{Cog} \operatorname{Imp}$ & -0.493 & $-1.000,0.013$ & 0.056 & -0.331 & $-0.835,0.172$ & 0.197 & -0.301 & $-0.809,0.280$ & 0.246 & -0.408 & $-1.806,-0.011$ & 0.044 \\
\hline Prefrail with $\operatorname{Cog} \operatorname{Imp}$ & -0.842 & $-1.542,-0.143$ & 0.018 & -0.642 & $-1.336,0.052$ & 0.070 & -0.515 & $-1.229,0.198$ & 0.157 & -0.681 & $-1.241,-0.121$ & 0.017 \\
\hline Frail without $\operatorname{Cog}$ Imp & -2.001 & $-2.847,-1.115$ & 0.000 & -1.535 & $-2.391,-0.679$ & 0.000 & -1.362 & $-2.240,-0.484$ & 0.002 & -1.104 & $-1.799,-0.409$ & 0.002 \\
\hline Frail with $\operatorname{Cog} \operatorname{Imp}$ & -1.378 & $-2.499,-0.258$ & 0.016 & -0.859 & $-1.984,0.266$ & 0.135 & -0.712 & $-1.849,0.425$ & 0.220 & -0.509 & $-1.397,0.379$ & 0.261 \\
\hline
\end{tabular}

Note: CI, 95\% confidence intervals; Cog Imp, cognitive impairment; Ref., reference. Model 1: adjusted for age (years; continuous). Model 2: adjusted for age (years; continuous), alcohol consumption (never, ever), and years of education (0-6, $\geq 7$ ). Model 3: adjusted for age (years; continuous), alcohol consumption (never, ever), years of education ( $0-6, \geq 7)$, comorbidities $(0, \geq 1)$, medications $(0,1-4, \geq 5)$, and body mass index $(\leq 23,23-24.9,25-26.9, \geq 27)$. 


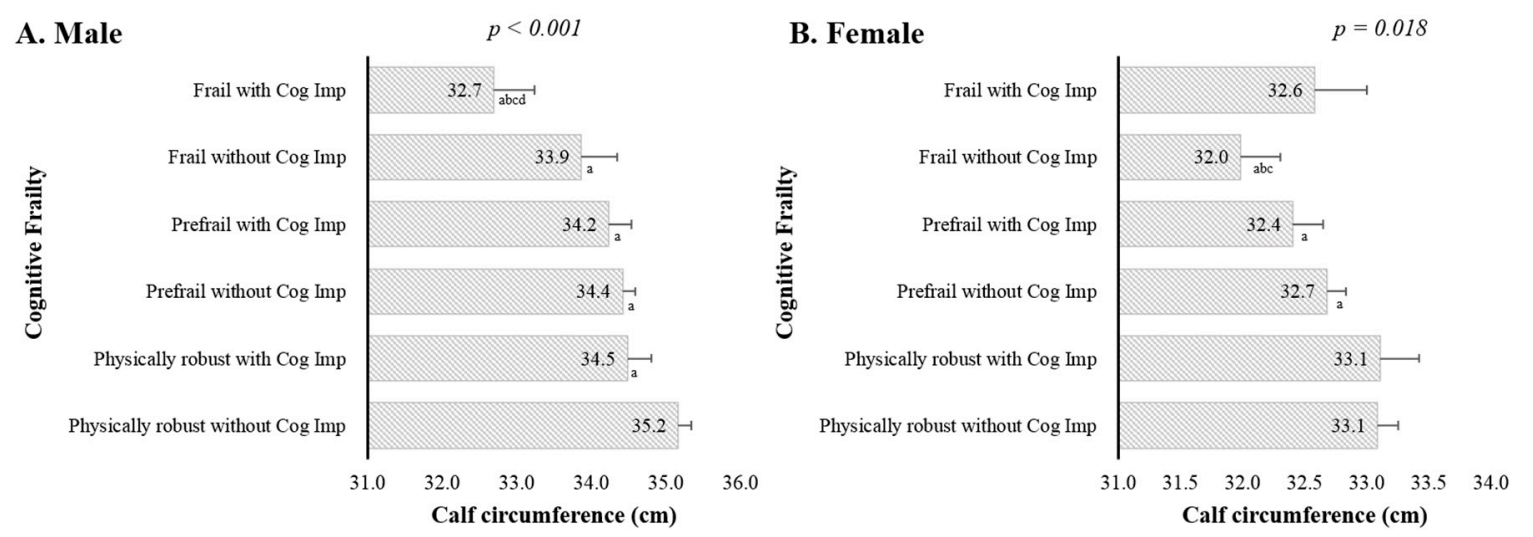

Figure 2. (A) male; (B) female. Geometric mean ( \pm SE) calf circumference according to physical frailty and cognitive impairment status. The multivariate analyses were adjusted for Model 3 (age, alcohol, years of education, comorbidity, medication, and body mass index). $p$-value from test for linear trend. $\mathrm{SE}=$ standard error; $\operatorname{Cog} \operatorname{Imp}=$ cognitive impairment. Differences determined by post-hoc analysis were considered significant at $p<0.05$. ${ }^{\text {a }}$ Significantly different from physically robust without $\operatorname{Cog} \operatorname{Imp}$ group. ${ }^{b}$ Significantly different from physically robust with Cog Imp group. ${ }^{c}$ Significantly different from prefrail without Cog Imp group. ${ }^{\mathrm{d}}$ Significantly different from prefrail with Cog Imp group.

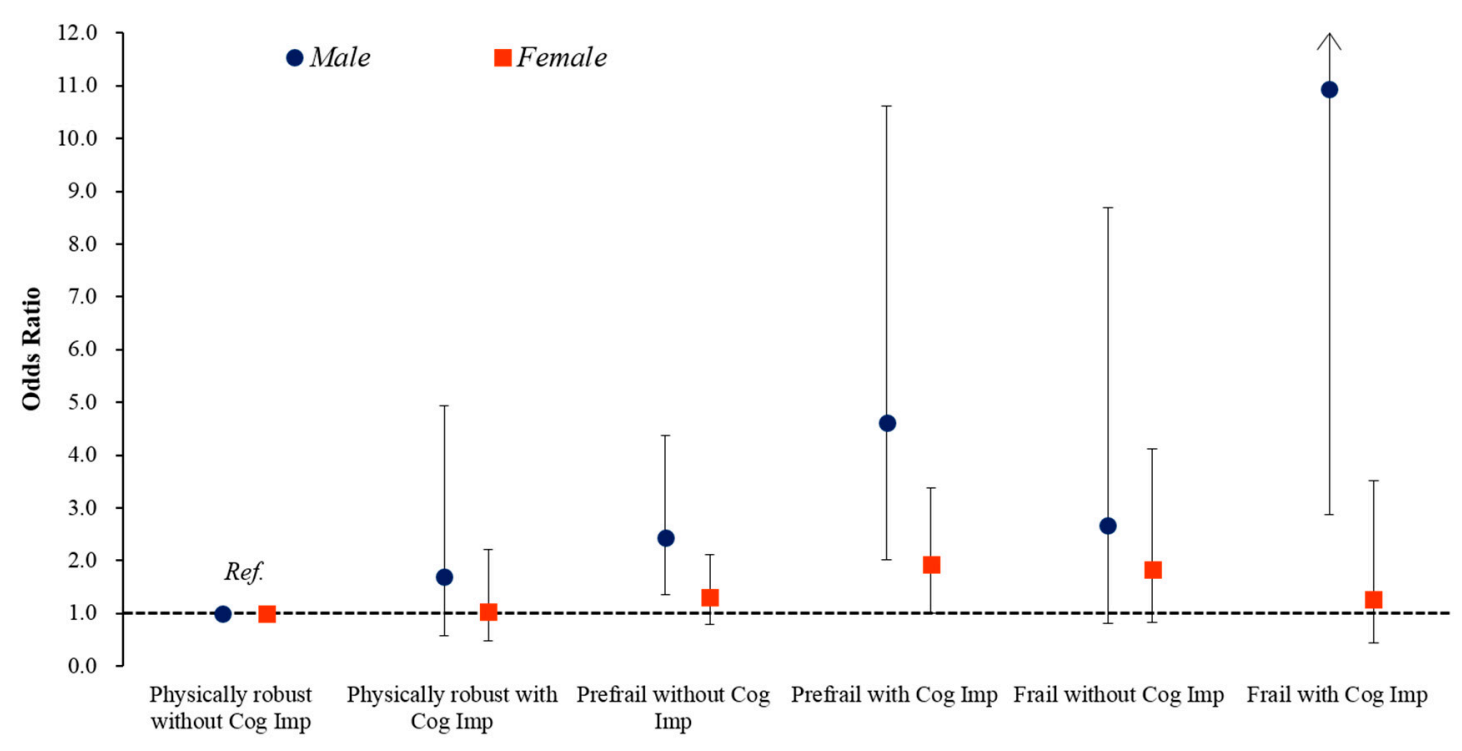

Figure 3. Association between low calf circumference $(<32 \mathrm{~cm})$ and cognitive frailty status. Odds ratios ( $95 \%$ confidence intervals) were calculated by Firth's penalized likelihood of logistic regression after adjustment for age, alcohol, years of education, comorbidity, medication, and body mass index.

\section{Discussion}

In our community-based sample of older adults, we found a significant decreasing trend in calf circumference across the six study groups, ranging from the physically robust without cognitive impairment to the physically frail with cognitive impairment group, in men only. Furthermore, we demonstrated that low calf circumference can be used to screen for prefrailty, cognitive prefrailty, and cognitive frailty in men, but not in women. Our findings underscore the utility of combining physical frailty and cognitive impairment indices and imply that calf circumference can serve as a simple screening tool for potential early detection of elderly men at risk of cognitive frailty. In the present analyses, no association was found between low calf circumference and cognitive frailty among elderly women. To the best of our knowledge, this is the first study to investigate the relationship between calf circumference and cognitive frailty in community-dwelling older adults. 
The prevalence of co-existing physical frailty and cognitive impairment was approximately $3.4 \%$ among our sample of older Koreans, and was significantly higher in women than in men. Similar to these results, Shimada et al. reported a prevalence of physical frailty (according to the Fried Frailty Index) accompanied by mild cognitive impairment of $2.7 \%$ in a sample of 5104 Japanese community-dwelling elderly adults (aged $\geq 65$ years) [38]. However, in a systematic review, the prevalence of cognitive frailty, ranging from $1.0 \%$ to $39.7 \%$, depended on the population characteristics and definition used [39]. Cognitive frailty has been associated with high risks of disability and dementia, poor quality of life, and death in the community setting [3,8,40-42].

Calf circumference has been used as a simple proxy marker for skeletal muscle mass, sarcopenia, and nutritional status in clinical and community settings [14-18]. Furthermore, it has been shown to be effective in predicting subsequent disability, an emerging need for care, and mortality in older adults [43-45]. On analyzing a representative sample of older US adults (age range: 60-84 years) according to four anthropometric parameters (waist, arm, thigh, and calf circumferences), only calf circumference showed a significant (inverse) association with disability [44]. In the Taiwan Longitudinal Survey on Aging Study [45], calf circumference was a better predictor of an emerging need for care in older adults compared to BMI.

In our study, we found a significant association between calf circumference and physical frailty in both sexes, consistent with the findings of the Aging and Longevity Study [19]. In that study, after adjusting for potential confounders, the Fried Frailty Index was significantly lower in participants with a larger versus smaller calf circumference (1.66 vs. 2.17 , respectively) among a population of older adults (aged $\geq 80$ years). In another study, there was a close relationship between calf circumference and ASM [46]. Mechanistically, muscle mass atrophy is a major factor in the pathogenesis of frailty [47], whereas calf circumference is a marker of the reduced muscle mass that characterizes sarcopenia and is related to physical function $[14,15]$. Nutritional status may be poor in frail older adults [48], and calf circumference can serve as a proxy for monitoring nutritional status in older populations [49]. In summary, calf circumference may be useful for monitoring physical frailty in older community-dwelling adults in clinical and research settings.

Age-related decline in cognitive function is a key aspect of dementia, and is also important for early detection of cognitive impairment in older community-dwelling adults. Body composition may serve as a modifiable risk factor of cognitive impairment. In a meta-analysis, sarcopenia and low muscle mass were independently associated with cognitive impairment [12]. Nishiguchi et al. found that both cognitive impairment and sarcopenia were associated with frailty [50]. In a population-based cross-sectional study, increased muscle mass and a lower level of adipose tissue were associated with superior cognitive function in older adults aged $\geq 60$ years [20]. Specifically, these studies showed that a larger calf circumference was associated with superior global cognition, memory, attention, information processing, and verbal learning. The present study showed that low calf circumference was most strongly related to cognitive impairment in community-dwelling Korean elderly men. Unlike in men, the lowest calf circumference was not seen in the cognitively frail group of women. Furthermore, in our subsequent analysis, there was no association between low calf circumference $(<31 \mathrm{~cm})$ and cognitive frailty status in women (data not shown). In a previous study, a calf circumference of $31 \mathrm{~cm}$ was considered the cut-off point for low muscle mass in community-dwelling older women [46]. One possible explanation for this difference is that the strength of the relationship between cognitive function and (age-related) decreased muscle mass differs between men and women: cognitive frailty was associated with low muscle mass $(87.5 \%)$ in men, but not in women $(32.0 \%)$. This is in line with previous studies regarding sex-specific differences in the relationship between low muscle mass and impaired cognitive functioning [51,52]. A large epidemiological study reported no association between low muscle mass and cognitive impairment after adjusting for potential confounders in a sample of 3025 women aged $\geq 75$ years [51]. A Chinese study showed a significant relationship between cognitive impairment and low muscle mass (whole-body muscle mass and ASM) among community-dwelling men aged $\geq 65$ years, but not in women [52]. Similarly, inverse associations between fat and dementia 
in women may be related to the regulation of female hormones [11]. In that study, higher percentages of subcutaneous abdominal and thigh fat were associated with a lower likelihood of dementia in women only. Longitudinal studies are warranted to elucidate further the mechanism underlying the association between calf circumference and cognitive impairment.

Our study had some limitations. First, its cross-sectional design did not allow evaluation of any cause-effect relationship between calf circumference and cognitive frailty. Second, our participants were community-dwelling older adults, recruited in a research setting; therefore, our results may not be generalized to other settings and populations. To confirm further the association between calf circumference and cognitive impairment, prospective studies are needed. We plan to conduct a follow-up longitudinal study on the predictive validity of calf circumference for development of cognitive frailty. Despite some limitations, this study benefitted from including a nationally representative sample of community-dwelling elderly Korean adults.

\section{Conclusions}

This study showed that low calf circumference was strongly related to cognitive frailty in community-dwelling Korean elderly men, for whom calf circumference can therefore be used as an indicator of cognitive frailty. Longitudinal studies are needed to determine the association between calf circumference and cognitive frailty over longer periods and sex-based differences.

Author Contributions: Conceptualization, C.W.W.; methodology, D.Y.S. and M.K.; formal analysis, D.Y.S. and M.J.J.; investigation and data curation, D.Y.S., M.J.J., M.K. and C.W.W.; original draft preparation, M.J.J. and M.K.; manuscript review and editing, C.W.W., M.K., M.J.J. and J.Y.; project administration, C.W.W.

Funding: This research was funded by the Ministry of Health and Welfare, Republic of Korea (grant number: HI15C3153).

Acknowledgments: We are deeply grateful to the study participants and the staff of the Korean Frailty and Aging Cohort Study for their cooperation.

Conflicts of Interest: The authors declare no conflicts of interest.

\section{References}

1. Fried, L.P.; Tangen, C.M.; Walston, J.; Newman, A.B.; Hirsch, C.; Gottdiener, J.; Seeman, T.; Tracy, R.; Kop, W.J.; Burke, G.; et al. Frailty in older adults: Evidence for a phenotype. J. Gerontol. Ser. A Biol. Sci. Med. Sci. 2001, 56, M146-M156. [CrossRef]

2. Walston, J.; Hadley, E.C.; Ferrucci, L.; Guralnik, J.M.; Newman, A.B.; Studenski, S.A.; Ershler, W.B.; Harris, T.; Fried, L.P. Research agenda for frailty in older adults: Toward a better understanding of physiology and etiology: Summary from the american geriatrics society/national institute on aging research conference on frailty in older adults. J. Am. Geriatr. Soc. 2006, 54, 991-1001. [CrossRef] [PubMed]

3. Feng, L.; Zin Nyunt, M.S.; Gao, Q.; Feng, L.; Yap, K.B.; Ng, T.P. Cognitive frailty and adverse health outcomes: Findings from the singapore longitudinal ageing studies (SLAS). J. Am. Med. Dir. Assoc. 2017, 18, $252-258$. [CrossRef] [PubMed]

4. Kelaiditi, E.; Cesari, M.; Canevelli, M.; van Kan, G.A.; Ousset, P.J.; Gillette-Guyonnet, S.; Ritz, P.; Duveau, F.; Soto, M.E.; Provencher, V.; et al. Cognitive frailty: Rational and definition from an (IANA/IAGG) international consensus group. J. Nutr. Health Aging 2013, 17, 726-734. [CrossRef] [PubMed]

5. Rodriguez-Manas, L.; Feart, C.; Mann, G.; Vina, J.; Chatterji, S.; Chodzko-Zajko, W.; Gonzalez-Colaco Harmand, M.; Bergman, H.; Carcaillon, L.; Nicholson, C.; et al. Searching for an operational definition of frailty: A delphi method based consensus statement: The frailty operative definition-consensus conference project. J. Gerontol. Ser. Biol. Sci. Med. Sci. 2013, 68, 62-67. [CrossRef] [PubMed]

6. Gross, A.L.; Xue, Q.L.; Bandeen-Roche, K.; Fried, L.P.; Varadhan, R.; McAdams-DeMarco, M.A.; Walston, J.; Carlson, M.C. Declines and impairment in executive function predict onset of physical frailty. J. Gerontol. Ser. Biol. Sci. Med. Sci. 2016, 71, 1624-1630. [CrossRef] [PubMed] 
7. Halil, M.; Cemal Kizilarslanoglu, M.; Emin Kuyumcu, M.; Yesil, Y.; Cruz Jentoft, A.J. Cognitive aspects of frailty: Mechanisms behind the link between frailty and cognitive impairment. J. Nutr. Health Aging 2015, 19, 276-283. [CrossRef] [PubMed]

8. $\quad$ Feng, L.; Nyunt, M.S.Z.; Gao, Q.; Feng, L.; Lee, T.S.; Tsoi, T.; Chong, M.S.; Lim, W.S.; Collinson, S.; Yap, P.; et al. Physical frailty, cognitive impairment, and the risk of neurocognitive disorder in the Singapore longitudinal ageing studies. J. Gerontol. Ser. A Biol. Sci. Med. Sci. 2017, 72, 369-395. [CrossRef] [PubMed]

9. Solfrizzi, V.; Scafato, E.; Seripa, D.; Lozupone, M.; Imbimbo, B.P.; D’Amato, A.; Tortelli, R.; Schilardi, A.; Galluzzo, L.; Gandin, C.; et al. Reversible cognitive frailty, dementia, and all-cause mortality. The Italian longitudinal study on aging. J. Am. Med. Dir. Assoc. 2017, 18, 89e81-89e88. [CrossRef] [PubMed]

10. Cruz-Jentoft, A.J.; Baeyens, J.P.; Bauer, J.M.; Boirie, Y.; Cederholm, T.; Landi, F.; Martin, F.C.; Michel, J.P.; Rolland, Y.; Schneider, S.M.; et al. Sarcopenia: European consensus on definition and diagnosis: Report of the european working group on sarcopenia in older people. Age Ageing 2010, 39, 412-423. [CrossRef] [PubMed]

11. Spauwen, P.J.; Murphy, R.A.; Jonsson, P.V.; Sigurdsson, S.; Garcia, M.E.; Eiriksdottir, G.; van Boxtel, M.P.; Lopez, O.L.; Gudnason, V.; Harris, T.B.; et al. Associations of fat and muscle tissue with cognitive status in older adults: The ages-reykjavik study. Age Ageing 2017, 46, 250-257. [CrossRef] [PubMed]

12. Chang, K.V.; Hsu, T.H.; Wu, W.T.; Huang, K.C.; Han, D.S. Association between sarcopenia and cognitive impairment: A systematic review and meta-analysis. J. Am. Med. Dir. Assoc. 2016, 17, 1164.e7-1164.e15. [CrossRef] [PubMed]

13. Cesari, M.; Leeuwenburgh, C.; Lauretani, F.; Onder, G.; Bandinelli, S.; Maraldi, C.; Guralnik, J.M.; Pahor, M.; Ferrucci, L. Frailty syndrome and skeletal muscle: Results from the invecchiare in chianti study. Am. J. Clin. Nutr. 2006, 83, 1142-1148. [CrossRef] [PubMed]

14. Kawakami, R.; Murakami, H.; Sanada, K.; Tanaka, N.; Sawada, S.S.; Tabata, I.; Higuchi, M.; Miyachi, M. Calf circumference as a surrogate marker of muscle mass for diagnosing sarcopenia in Japanese men and women. Geriatr. Gerontol. Int. 2015, 15, 969-976. [CrossRef] [PubMed]

15. Kim, S.; Kim, M.; Lee, Y.; Kim, B.; Yoon, T.Y.; Won, C.W. Calf circumference as a simple screening marker for diagnosing sarcopenia in older korean adults: The korean frailty and aging cohort study (KFACS). J. Korean Med. Sci 2018, 33, e151. [CrossRef] [PubMed]

16. Maeda, K.; Koga, T.; Nasu, T.; Takaki, M.; Akagi, J. Predictive accuracy of calf circumference measurements to detect decreased skeletal muscle mass and European society for clinical nutrition and metabolism-defined malnutrition in hospitalized older patients. Ann. Nutr. Metab. 2017, 71, 10-15. [CrossRef] [PubMed]

17. Aparecida Leandro-Merhi, V.; Luiz Braga de Aquino, J.; Gonzaga Teixeira de Camargo, J. Agreement between body mass index, calf circumference, arm circumference, habitual energy intake and the mna in hospitalized elderly. J. Nutr. Health Aging 2012, 16, 128-132. [CrossRef] [PubMed]

18. Kim, H.; Suzuki, T.; Kim, M.; Kojima, N.; Yoshida, Y.; Hirano, H.; Saito, K.; Iwasa, H.; Shimada, H.; Hosoi, E.; et al. Incidence and predictors of sarcopenia onset in community-dwelling elderly japanese women: 4-year follow-up study. J. Am. Med. Dir. Assoc. 2015, 16, 85.e1-85.e8. [CrossRef] [PubMed]

19. Landi, F.; Onder, G.; Russo, A.; Liperoti, R.; Tosato, M.; Martone, A.M.; Capoluongo, E.; Bernabei, R. Calf circumference, frailty and physical performance among older adults living in the community. Clin. Nutr. 2014, 33, 539-544. [CrossRef] [PubMed]

20. Won, H.; Abdul Manaf, Z.; Mat Ludin, A.F.; Shahar, S. Wide range of body composition measures are associated with cognitive function in community-dwelling older adults. Geriatr. Gerontol. Int. 2017, 17, 554-560. [CrossRef] [PubMed]

21. Won, C.W.; Lee, Y.; Choi, J.; Kim, K.W.; Park, Y.; Park, H.; Oh, I.-H.; Ga, H.; Kim, Y.S.; Jang, H.C. Starting construction of frailty cohort for elderly and intervention study. Ann. Geriatr. Med. Res. 2016, 20, 114-117. [CrossRef]

22. Son, J.H.; Kim, S.Y.; Won, C.W.; Choi, H.R.; Kim, B.S.; Park, M.S. Physical frailty predicts medical expenses in community-dwelling, elderly patients: Three-year prospective findings from living profiles of older people surveys in korea. Eur. Geriatr. Med. 2015, 6, 412-416. [CrossRef]

23. Won, C.W.; Lee, Y.; Kim, S.; Yoo, J.; Kim, M.; Ng, T.P.; Kim, H.; Son, S.J. Modified criteria for diagnosing "cognitive frailty". Psychiatry Investig. 2018, 15, 839-842. [CrossRef] [PubMed] 
24. Lee, J.H.; Lee, K.U.; Lee, D.Y.; Kim, K.W.; Jhoo, J.H.; Kim, J.H.; Lee, K.H.; Kim, S.Y.; Han, S.H.; Woo, J.I. Development of the korean version of the consortium to establish a registry for alzheimer's disease assessment packet (CERAD-K): Clinical and neuropsychological assessment batteries. J. Gerontol. Ser. B Psychol. Sci. Soc. Sci. 2002, 57, P47-P53. [CrossRef]

25. Lee, D.Y.; Lee, K.U.; Lee, J.H.; Kim, K.W.; Jhoo, J.H.; Kim, S.Y.; Yoon, J.C.; Woo, S.I.; Ha, J.; Woo, J.I. A normative study of the cerad neuropsychological assessment battery in the Korean elderly. J. Int. Neuropsychol. Soc. 2004, 10, 72-81. [CrossRef] [PubMed]

26. Kim, T.H.; Huh, Y.; Choe, J.Y.; Jeong, J.W.; Park, J.H.; Lee, S.B.; Lee, J.J.; Jhoo, J.H.; Lee, D.Y.; Woo, J.I.; et al. Korean version of frontal assessment battery: Psychometric properties and normative data. Dement. Geriatr. Cognit. Disord. 2010, 29, 363-370. [CrossRef] [PubMed]

27. Aisen, P.S. Treatment for MCI: Is the evidence sufficient? Neurology 2008, 70, 2020-2021. [CrossRef] [PubMed]

28. Petersen, R.C.; Smith, G.E.; Waring, S.C.; Ivnik, R.J.; Tangalos, E.G.; Kokmen, E. Mild cognitive impairment: Clinical characterization and outcome. Arch. Neurol. 1999, 56, 303-308. [CrossRef] [PubMed]

29. Jessen, F.; Amariglio, R.E.; van Boxtel, M.; Breteler, M.; Ceccaldi, M.; Chetelat, G.; Dubois, B.; Dufouil, C.; Ellis, K.A.; van der Flier, W.M.; et al. A conceptual framework for research on subjective cognitive decline in preclinical Alzheimer's disease. Alzheimer's Dement. J. Alzheimer's Assoc. 2014, 10, 844-852. [CrossRef] [PubMed]

30. Dubois, B.; Feldman, H.H.; Jacova, C.; Cummings, J.L.; Dekosky, S.T.; Barberger-Gateau, P.; Delacourte, A.; Frisoni, G.; Fox, N.C.; Galasko, D.; et al. Revising the definition of Alzheimer's disease: A new lexicon. Lancet Neurol. 2010, 9, 1118-1127. [CrossRef]

31. Lee, W.J.; Peng, L.N.; Liang, C.K.; Loh, C.H.; Chen, L.K. Cognitive frailty predicting all-cause mortality among community-living older adults in Taiwan: A 4-year nationwide population-based cohort study. PLoS ONE 2018, 13, e0200447. [CrossRef] [PubMed]

32. Shimada, H.; Doi, T.; Lee, S.; Makizako, H.; Chen, L.K.; Arai, H. Cognitive frailty predicts incident dementia among community-dwelling older people. J. Clin. Med. 2018, 7, 250. [CrossRef] [PubMed]

33. Chen, L.K.; Liu, L.K.; Woo, J.; Assantachai, P.; Auyeung, T.W.; Bahyah, K.S.; Chou, M.Y.; Chen, L.Y.; Hsu, P.S.; Krairit, O.; et al. Sarcopenia in asia: Consensus report of the asian working group for sarcopenia. J. Am. Med. Dir. Assoc. 2014, 15, 95-101. [CrossRef] [PubMed]

34. Guralnik, J.M.; Simonsick, E.M.; Ferrucci, L.; Glynn, R.J.; Berkman, L.F.; Blazer, D.G.; Scherr, P.A.; Wallace, R.B. A short physical performance battery assessing lower extremity function: Association with self-reported disability and prediction of mortality and nursing home admission. J. Gerontol. 1994, 49, M85-M94. [CrossRef] [PubMed]

35. Guralnik, J.M.; Ferrucci, L.; Pieper, C.F.; Leveille, S.G.; Markides, K.S.; Ostir, G.V.; Studenski, S.; Berkman, L.F.; Wallace, R.B. Lower extremity function and subsequent disability: Consistency across studies, predictive models, and value of gait speed alone compared with the short physical performance battery. J. Gerontol. Ser. A Biol. Sci. Med. Sci. 2000, 55, M221-M231. [CrossRef]

36. Podsiadlo, D.; Richardson, S. The timed "up \& go": A test of basic functional mobility for frail elderly persons. J.Am. Geriatr. Soc. 1991, 39, 142-148. [PubMed]

37. Heinze, G.; Schemper, M. A solution to the problem of separation in logistic regression. Stat. Med. 2002, 21, 2409-2419. [CrossRef] [PubMed]

38. Shimada, H.; Makizako, H.; Doi, T.; Yoshida, D.; Tsutsumimoto, K.; Anan, Y.; Uemura, K.; Ito, T.; Lee, S.; Park, H.; et al. Combined prevalence of frailty and mild cognitive impairment in a population of elderly Japanese people. J. Am. Med. Dir. Assoc. 2013, 14, 518-524. [CrossRef] [PubMed]

39. Sugimoto, T.; Sakurai, T.; Ono, R.; Kimura, A.; Saji, N.; Niida, S.; Toba, K.; Chen, L.K.; Arai, H. Epidemiological and clinical significance of cognitive frailty: A mini review. Ageing Res. Rev. 2018, 44, 1-7. [CrossRef] [PubMed]

40. Solfrizzi, V.; Scafato, E.; Lozupone, M.; Seripa, D.; Giannini, M.; Sardone, R.; Bonfiglio, C.; Abbrescia, D.I.; Galluzzo, L.; Gandin, C.; et al. Additive role of a potentially reversible cognitive frailty model and inflammatory state on the risk of disability: The Italian longitudinal study on aging. Am. J. Geriatr. Psychiatry 2017, 25, 1236-1248. [CrossRef] [PubMed]

41. Shimada, H.; Makizako, H.; Lee, S.; Doi, T.; Lee, S.; Tsutsumimoto, K.; Harada, K.; Hotta, R.; Bae, S.; Nakakubo, S.; et al. Impact of cognitive frailty on daily activities in older persons. J. Nutr. Health Aging 2016, 20, 729-735. [CrossRef] [PubMed] 
42. St John, P.D.; Tyas, S.L.; Griffith, L.E.; Menec, V. The cumulative effect of frailty and cognition on mortality-results of a prospective cohort study. Int. Psychogeriatr. 2017, 29, 535-543. [CrossRef] [PubMed]

43. Tsai, A.C.; Chang, T.L. The effectiveness of BMI, calf circumference and mid-arm circumference in predicting subsequent mortality risk in elderly taiwanese. Br. J. Nutr. 2011, 105, 275-281. [CrossRef] [PubMed]

44. Sun, Y.S.; Kao, T.W.; Chang, Y.W.; Fang, W.H.; Wang, C.C.; Wu, L.W.; Yang, H.F.; Liaw, F.Y.; Chen, W.L. Calf circumference as a novel tool for risk of disability of the elderly population. Sci. Rep. 2017, 7, 16359. [CrossRef] [PubMed]

45. Hsu, W.C.; Tsai, A.C.; Wang, J.Y. Calf circumference is more effective than body mass index in predicting emerging care-need of older adults-Results of a national cohort study. Clin. Nutr. 2016, 35, 735-740. [CrossRef] [PubMed]

46. Rolland, Y.; Lauwers-Cances, V.; Cournot, M.; Nourhashemi, F.; Reynish, W.; Riviere, D.; Vellas, B.; Grandjean, H. Sarcopenia, calf circumference, and physical function of elderly women: A cross-sectional study. J. Am. Geriatr. Soc. 2003, 51, 1120-1124. [CrossRef] [PubMed]

47. Walston, J.; Fried, L.P. Frailty and the older man. Med. Clin. N. Am. 1999, 83, 1173-1194. [CrossRef]

48. Kim, J.; Lee, Y.; Won, C.W.; Lee, K.E.; Chon, D. Nutritional status and frailty in community-dwelling older korean adults: The korean frailty and aging cohort study. J. Nutr. Health Aging 2018, 22, 774-778. [CrossRef] [PubMed]

49. Portero-McLellan, K.C.; Staudt, C.; Silva, F.R.; Delbue Bernardi, J.L.; Baston Frenhani, P.; Leandro Mehri, V.A. The use of calf circumference measurement as an anthropometric tool to monitor nutritional status in elderly inpatients. J. Nutr. Health Aging 2010, 14, 266-270. [CrossRef] [PubMed]

50. Nishiguchi, S.; Yamada, M.; Fukutani, N.; Adachi, D.; Tashiro, Y.; Hotta, T.; Morino, S.; Shirooka, H.; Nozaki, Y.; Hirata, H.; et al. Differential association of frailty with cognitive decline and sarcopenia in community-dwelling older adults. J. Am. Med. Dir. Assoc. 2015, 16, 120-124. [CrossRef] [PubMed]

51. Abellan van Kan, G.; Cesari, M.; Gillette-Guyonnet, S.; Dupuy, C.; Nourhashemi, F.; Schott, A.M.; Beauchet, O.; Annweiler, C.; Vellas, B.; Rolland, Y. Sarcopenia and cognitive impairment in elderly women: Results from the epidos cohort. Age Ageing 2013, 42, 196-202. [CrossRef] [PubMed]

52. Auyeung, T.W.; Kwok, T.; Lee, J.; Leung, P.C.; Leung, J.; Woo, J. Functional decline in cognitive impairment-The relationship between physical and cognitive function. Neuroepidemiology 2008, 31, 167-173. [CrossRef] [PubMed] 